LBL -18302

DE85 002005

\title{
Geometric Hamiltonian Structures and Perturbation Theory*
}

\author{
Stephen Omohundro \\ Lawrence Berkeley Laboratory and Physics Dapartment \\ University of California \\ -Berkeley, CA 94720
}

\begin{abstract}
August 1984
Presented at the NRL-NSWC Workshop on

Local \& Global Methods of Dynamics, Naval Surface Weapons Center, S1lver

Spring, Maryland, July 23-26, 1984
\end{abstract}

\section{DISCLAIMER}

This report was prepared as an account of work sponsored by an agersy of the United States Government. Neither the United States Government nor any agency thereof, nor any of their employees, makes any warranty, express or implied, or ussumes any legal liability or responsibility for the accuracy, completeness, or usefulness of any information, apparatus, product, or process disclosed, or represents that its use would not infringe privately owned rights. Reference herein to any speciffe commercial product, process, or service by trade name, trademark, manufacturer, or otherwise does not necessarily constitute or imply its endorsement, recommendation, or favoring by the United States Government or any agency thereof. The views and opinions of authors expressed herein do rot necessarily state or reflect those of the Uisited States Government or any agency thereof.

*This work was supported by the Office of Basic Energy Sciences of the U.S. Department of Energy under Contract No. OE-ACO3-76SF00098. 
Geometric Hamiltonian Structures and Perturbation Theory $\dagger$

by Stephen Omohundro

Lawrence Berkeley Laboratory and Physics Department

University of California

Berkzley, CA 94720

\section{Introduction}

In this lecture we discuss the ideas in reference [25] intuitively and heuristically. That paper assumes a background in geometric mechanics and has detailed proofs. Here we will give the flavor of the structures and develop the needed background material. We will state the results and indicate why they are true without detailed preof. We begin with some introductory rernarks, discuss a geometric picture for non-singular perturbation theory, introduce the needed Hamiltonian mechanics including the crucial process of reduction in the presence of symmetry, describe the Hamiltncian structure of non-siugular perturbation theory, and close with some discussion of these ideas in connection witb the method of averaging.

Dr. Deprit's lecture has described the intimate historical coanections between perturbation theory and Hamiltonian mechanics. It is of interest to list the seminal ideas that form the background of the present work. In 1808 Lagrange introduced the description of the dynamics of celestial bodies in terms of what we today call Hamilton's equations ${ }^{1,2}$. His motivation was the reduction of the enormous labor involved in a straightforward pertiorbation analysis, which required tedious computations to be performed on each component of the dynamical vector field, to maripulations of a single function: the Hamiltonian. The description in terms of Lagrange brackets led to several other benefits: Lagrange showed that the value of the Hamiltonian and the structure of the brackets were both invariant under the dynamics, leading to a useful check of the complex calculations (which at that time were done by hand). In addition, he was able to show that the invariance of the Hamiltonian could be used to prove stability of certain equilibria. As the century progressed, Hamiltonian mechanics was refined and the connections with variational principles and optics were made. By the turn of the century Poincare ${ }^{3}$ had developed very powerful Hamiltonian perturbation methods utilizing generating functions, introduced the notion of asymptotic expansion, and begun the geometric and topological approach to dynamics. In 1918 Emmy Noether ${ }^{4}$ made the connection between symmetries and conserved quantities. The development of quantum mechanics rested heavily on the Hamiltonian framework ${ }^{5}$ by analogy with optics and served to put it firmly at the center of the modern formulation of fundamental physics ${ }^{6}$. During the 19ô0's the coordinate free description of Hamiltonian structures in terms of symplectic geometry was developed ${ }^{7,8}$. About this time the Hamiltonian method of Lie transforms greatly simplified Hamiltonian perturbation thcory ${ }^{9}$. The 1970's saw enormous

$\dagger$ This work was supported by the Office of Basic Energy Sciences of the U.S. Department of Energy under Contract No. DE-AC03-76SF00098. 
developments in the geometric approach to mechanics and largely as a result of these, an ever wider range of physical systems have been described in Hamiltonian terms. Some examples are: quantum mechanics ${ }^{10}$, fluid mechanics ${ }^{11,12,13}$, Maxwell's equations ${ }^{15,16}$, the Maxwell-Vlasov and Poisson-Vlasov ${ }^{14,15}$ equations of plasma physics, elasticity theory ${ }^{17,18}$, general relativity ${ }^{18}$, magnetohydrodynamics ${ }^{11,17}$, multi-fluid plasmas ${ }^{17,19}$, chromohydrodynamics ${ }^{20}$, superfluids and superconductors $^{21}$, the Korteweg de Vries equation ${ }^{22}$, etc. These developments have shed light on the underlying symmetry structure of these theories, have yielded improved stability results based on Arnold s stability method ${ }^{23}$, and have given insight into the reasons for the integrability of certain systems ${ }^{24}$.

For the most part, however, these structures describe fundamental underlying models in the various fields. In actual applications we almost always make numerous approximations which may or may not respect the underlying Hamiltonian structure. It is folklore within the particle physics community and elsewhere that perturbation methods which respect the underlying symmetries and conservation laws yield much better approximations to the actual system than thase which do not. It is of interest, then, to try to do perturbation theory within the Hamiltonian framework and to obtain structures relevant to the approximate system. One may thus hope to understand thá relation between the structures of systems which are limiting cases of known systems (eg. does the KdV Poisson bracket arise naturally from that of the Boussinesq equations?) $)^{31}$. The history of Hamiltonian mechanics is inextricably tied to perturbation methods. For the most part, though, the Hamiltonian structure was used to simplify the perturbation method and the geometric structure of the perturbation method itself was not explored. We have found in several examples that taking this structure into account leads to simplifications (as in the problem of guiding center motion discussed lates in this paper) and to deeper insight into the approximate system (as in modulational equations for waves in the eikonal limit) ${ }^{26}$.

We have therefore been engaged in a program of investigating the Hamiltonian structure of the various perturbation theories used in practice. In this paper we describe the geornetry of a Hamiltonian structure for non-singular perturbation theory applied to Hamiltonian systems on symplectic manifolds and the connection with singular perturbation techniques based on the method of averaging.

\section{Geometric Perturbation Theory}

The modern setting for describing an evolving system is a dynamical system. The state of the system is represented by a point in a manifold $M$. A manifold is a space which locally looks like Euclidean space and in which there is a notion of derivative. Globally a manifold may be connected together in a funny way, as in a sphere or a torus. If you know where you are, the dywamics tells you where you're going. Thus dynamics is represented by a vector field on the manifold of states. A dynamical system is a manifold with a vector field defined on it. In coordinates the dynamics gives a set of first order O.D.E.'s, one for each coordinate. Typical dynamical systems with state spaces of three dimensions or greater have chaotic dynamics with extremely complicated 
trajectories for which one can prove there is no closed form exact description. If the dynamics simplifies then there is usually some physically relevant special feature such as a symmetry which causes the simplification.

In important physical applications, we often find ouselves close to a system which simplifies and we are interested in the effect of our deviation from it. We express this deviation in terms of the small parameter $\epsilon$. If we are given dynamics in the form

$$
\dot{x}=X_{0}+\epsilon X_{1}+\frac{\epsilon^{3}}{2} X_{2}+\ldots
$$

in terms of the vector fields $X_{i}$ with initial conditions described by $x(\epsilon, t=0)=y(\epsilon)$, we may attempt tc express the solution in an asymptotic series in $\epsilon$ :

$$
x(t)=x_{0}(t)+\epsilon x_{1}(t)+\frac{\epsilon^{2}}{2} x_{2}(t)+\ldots
$$

Choosing coordinates $x^{a}(1 \leq a \leq N)$ in a local patch and plugging this assumed asymptotic form into the equation of motion gives:

$$
\begin{aligned}
\dot{x}_{0}^{a}+\epsilon \dot{x}_{1}^{a}+\frac{\epsilon^{2}}{2 !} \dot{x}_{2}^{a}+\ldots= & X_{0}^{a}\left(x_{0}+\epsilon x_{1}+\frac{\epsilon^{2}}{2 !} x_{2}+\ldots\right)+ \\
& +\epsilon X_{1}^{a}\left(x_{0}+\epsilon x_{1}+\frac{\epsilon^{2}}{2 !} x_{2}+\ldots\right)+ \\
& +\frac{\epsilon^{2}}{2 !} X_{2}^{a}\left(x_{0}+\epsilon x_{1}+\frac{\epsilon^{2}}{2 !} x_{2}+\ldots\right)+\ldots
\end{aligned}
$$

Since asymptotic expansioas are unique, we may equate coefficients of equal powers of $\epsilon$ to get equations for $x_{0}, x_{1}, \ldots$ :

$$
\begin{aligned}
\dot{x}_{0}^{a} & =X_{0}^{a}\left(x_{0}\right) \\
\dot{x}_{1}^{a} & =\sum_{b=1}^{N} \frac{\partial X_{0}^{a}}{\partial x^{b}}\left(x_{0}\right) \cdot x_{1}^{b}+X_{1}^{a}\left(x_{0}\right) \\
\dot{x}_{2}^{a} & =\sum_{b, c=1}^{N} \frac{\partial^{2} X_{0}^{a}}{\partial x^{b} \partial x^{c}}\left(x_{0}\right) x_{1}^{b} x_{1}^{c}+\sum_{b=1}^{N} \frac{\partial X_{0}^{a}}{\partial x^{b}}\left(x_{0}\right) \cdot x_{2}^{b} \\
& +2 \sum_{b=1}^{N} \frac{\partial X_{1}^{a}}{\partial x^{b}}\left(x_{0}\right) \cdot x_{1}^{b}+X_{2}^{a}\left(x_{0}\right)
\end{aligned}
$$

If $y(\epsilon)=y_{0}+\epsilon y_{1}+\frac{\epsilon^{2}}{2} y_{2}+\ldots$ is an asymptotic expansion for the initial condition $y(\epsilon)$, then the initial conditions for these equations are: $x_{0}(t=0)=y_{0}, x_{1}(t=0)=y_{1}, \ldots$

These equations immediately raise a number of questions. They are defined in terms of physically irrelevant coordinates; is the perturbation structure independent of these coordinates? If the original equations are Hamiltonian are these equations? In $J$ th order perturbation theory, how are we to interpret thiz evolution of many variables $x_{0}, x_{1}, \ldots, x_{J}$ ? The goal of this work is to answer these questions. 
Let us turn to the geometric interpretation of these equations. It is easiest to understand the first order perturbation equations:

$$
\begin{aligned}
& \dot{x}_{0}^{a}=X_{0}^{a}\left(x_{0}\right) \\
& \dot{x}_{1}^{a}=\sum_{b=1}^{N} \frac{\partial X_{0}^{a}}{\partial x^{b}}\left(x_{0}\right): x_{1}^{b}+X_{1}^{a}\left(x_{0}\right) \\
& x_{0}(t=0)=y_{0} \quad x_{1}(t=0)=y_{1} .
\end{aligned}
$$

We would like to determine the geometric nature of the quantities $x_{0}$ and $x_{1}$. To understand what we mean by this let us recall the relationship between geometric quantities and coordinates. A function on a manifold is an intrinsically defined thing, it assigns a real number to each point of the manifold. A coordinate system on a region of an $N$ dimensional manifold is a collection of $N$ real valued functions $x^{1}, \ldots, x^{N}$ defined on that region, whose differentials are linearly independent at each point. In these coordinates, the gradient of a function is a collection of $N$ numbers, the derivatives with respect to each of the $x^{a}$. Geometrically, however it is wrong to think of these as just real numbers because they change if we change our coordinate system. For example, if we choose coordinates whose values at each point of the region are twice those of $x^{1}, \ldots, x^{N}$ then the components of the gradient of a function are halved. We introduce a geometric object whose relationship to the manifold at a given point is like that of the gradient of a function and we call it a covector or one-form. The collection of all covectors at a point is defined to be the cotangent space at that point and the collection of all cotangent spaces taken together form the cotangent bundle. Similarly the components of a vector at a point double with the coordinates. All vectors at a point taken together form the tangent space at that point and all tangent spaces taken together form the tangent bundle $T M$ of $M$. Vectors and covectors are thus different objects when we consider nore than one coordinate system, even though they both have $N$ components in any given system. Our interest here will be to find out whether the quantities $x_{0}^{a}, \ldots, x_{J}^{\alpha}$ for $1 \leq a \leq N$ have any geometric structure that is independent of a given coordinate system.

Intuitively, the first order quantity $x_{1}$ represents a small deviation from the unperturbed quantity $x_{0}$. Because $x_{0}$ can vary over the whole manifold $M$, we expect it to represent a point in the manifold. As $\epsilon$ gets smaller $x_{0}+\epsilon x_{1}$ approaches the point $x_{0}$ and $x_{1}$ measures the first order rate of approach to $\tau_{0}$. Two different paths in the manifold approaching the point $x_{0}$ as $\epsilon$ approaches zero have the same $x_{1}$ if and only if they are tangent at $x_{0}$. This, however, is the defining criterion for a vector at the point $x_{0}$. We thus expect $x_{1}$ to lie in the tangent space to $M$ over the point $x_{0}$. The $x_{0}, x_{1}$ dynamics then takes place on the tangent bundle $T M$. We will describe this dynamics on $T M$ intrinsically in terms of vector fields derived from $X(\epsilon)$ on $M$.

The solution of a system of O.D.E.'s tells us the state at each time $t$ of a system which began with each initial condition. Geometrically, this is a mapping of $M$ to itself for each $t$. If the solution doesn't run off of the manifold, then the uniqueness and smoothness of solutions with given intial conditions tells us that this map is a diffeomorphism (i.e. a smooth, 1-1, onto map with smooth inversc). This one-paraneter family of diffeomorphisms labelled by $t$ is called the flow of the dynamical vector field. As $\epsilon$ varies, the corresponding flows of $X(\epsilon)$ will vary. 
Perturbation theory describes that variation. Any time we have a mapping $f$ from one manifold to another, we may define its differential $T f$. This is a map that takes the tangent bundle of the first manifold to the tangent bundle of the second. It describes how infinitesimal perturbations at a point are sent to infinitesimal perturbations at the image point. In coordinates, it acts on the tangent space at a point via the Jacobian matrix of $f$ at that point.

Through abuse of notation, let us denote the flow of the unperturbed vector field $X_{0}$ by $x_{0}(t) . x_{0}\left(t, y_{0}\right)$ is the point to which $y_{0}$ has flowed in time $t$ under $\boldsymbol{X}_{0}$. A small perturbation in $M$ from a given orbit will evolve under $X_{0}$ according to the derivative of this flow $T x_{0}(t)$. This is a flow on the manifold $T M$, and the vector field of which it is the flow may be written:

$$
\left.\tilde{X}_{0} \equiv \frac{d}{d t}\right|_{t=0} T x_{0}(t)
$$

$\tilde{X}_{0}$ is a vector field on $T M$ defined without recourse to coordinates that represents the effect of the unperturbed flow on perturbed orbits. In coordinates $\tilde{X}_{0}$ has components:

$$
\begin{aligned}
& \dot{x}_{0}^{a}=X_{0}^{a}\left(x_{0}\right) \\
& \dot{x}_{1}^{a}=\sum_{b=1}^{N} \frac{\partial X_{0}^{a}}{\partial x^{b}}\left(x_{0}\right) \cdot x_{1}^{b} .
\end{aligned}
$$

This dynamics is exactly that part of the perturbation dynamics (5) which depends on $X_{0}$. The part which depends on $X_{1}$ may also be defined intrinsically. If we define:

$$
\left.\tilde{X}_{1}(x, v) \equiv \frac{d}{d t}\right|_{t=0}\left(v+t X_{1}(x)\right),
$$

then the entire first order perturbation dynamics on $T M$ is given by

$$
\tilde{X}_{0}+\tilde{X}_{1}
$$

\section{The Geometry of Jth Order Perturbation Theory}

We have succeeded in finding a geometric, coordinate-free interpretation for first order perturbation theory. We now would like to extend this to higher orders. The geometric object that arises is called a jet. To understand tr.s setting, we discuss a number of relevant spaces.

How are we to think of the exact equation for the evolution of an $\epsilon$ dependent point $x(\epsilon)$ under $\epsilon$ dependent evolution equations $\dot{x}(\epsilon)=X(\epsilon, x)$ with $\epsilon$ dependent initial conditions $y(\epsilon)$ ? It is useful to think of the $\epsilon$ dependent point $x(\epsilon)$ as a curve in the space $I \times M$, where $I$ is the interval (say $[0,1 \mid)$ in which $\epsilon$ takes its values. If we think of $x(\varepsilon)$ as a map from $I$ to $M$, then the curve is the graph of this map. The dynamical vector field $X(\epsilon)$ naturally lives on $I \times M$ and its $I$ component is zero everywhere. The flow of $X(c)$ on $I \times M$ takes paths to paths by letting each point of a path move with the flow. Our initial conditions are represented by paths (if they are 
independent of $\epsilon$ then they are straight lines). The true dynamics takes paths to paths. Even if the intial conditions are $\epsilon$ independent, the dynamics bends the path over. Thus we really should think of our dynamics as living on the infinite dimensional path space:

$$
P_{1} M \equiv\{\text { space of all paths } p: I \rightarrow I \times M \text { of the form } p: \epsilon \mapsto(\epsilon, x(\epsilon))\}
$$

where as before $I=[0,1]$.

This projects naturally onto:

$$
P_{0} M \equiv\left\{\text { equivalence classes in } P_{1} M \text { where } p_{1} \sim p_{2} \text { iff } p_{1}(0)=p_{2}(0)\right\} .
$$

$P_{0} M$ is naturally isomorphic to $M$ and represents the domain of the unperturbed dynamics. The equivalence classes forget all perturbation information and only remember behavior at $\epsilon=0$. We are interested in spaces through which this projection of real to unperturbed dynamics factors. Perturbation theory tries to study behavior infinitesimally close to $\epsilon=0$ without actually getting there. For each $0 \leq \alpha \leq 1$ we may define:

$$
P_{\alpha} M \equiv\left\{\text { equivalence classes in } P_{1} M \text { where } p_{1} \sim p_{2} \text { iff } p_{1}(\epsilon)=p_{2}(\epsilon) \forall 0 \leq \epsilon \leq \alpha\right\} .
$$

These allow us to consider more and more restricted domains of $\epsilon$, but there is always a continuum of $\epsilon$ 's to traverse before reaching $\epsilon=0$. For each $1 \geq \alpha_{1} \geq \alpha_{2} \geq 0$ we have the natural maps:

$$
P_{1} M \rightarrow P_{\alpha_{1}} M \rightarrow P_{\alpha_{2}} M \rightarrow P_{0} M
$$

We are interested in structure between even the smallest $P_{\alpha} M$ with $\alpha \neq 0$ and $P_{0} M$. We may introduce germs of paths:

$$
\begin{array}{r}
G M \equiv\left\{\text { equivalence classes in } P_{1} M \text { where } p_{1} \sim p_{2}\right. \text { iff } \\
\left.\exists \alpha>0 \text { such that } p_{1}(\epsilon)=p_{2}(\epsilon) \forall 0 \leq \epsilon \leq \alpha\right\}
\end{array}
$$

and for any $\alpha>0$ we have $P_{\alpha} M \rightarrow G M \rightarrow P_{0} M$. The germs capture behavior closer to $\epsilon=0$ than any given $\epsilon$, but still contain much more information than perturbation theory gives us (germs depend on features of function in a little neighborhood that are not captured in a Taylor series.)

Finally we may introduce spaces of jets of paths at $\epsilon=0$ with integer $1 \leq J \leq \infty$ :

$$
\begin{aligned}
J M \equiv\{ & \left\{\text { equivalence classe: } 3 \text { in } P_{1} M \text { where } p_{1} \sim p_{2}\right. \text { iff } \\
& \forall C^{\infty} \text { functions } f \text { on } I \times M \text { we have : } \\
& \left.\left.\frac{\partial^{i}}{\partial \epsilon^{i}}\right|_{\epsilon=0} f\left(p_{1}(c)\right)=\left.\frac{\partial^{i}}{\partial \epsilon^{i}}\right|_{\epsilon=0} f\left(p_{2}(\epsilon)\right) \quad \text { for } 0 \leq i \leq J\right\} .
\end{aligned}
$$


Thus the space of $J$-jets gives the first $J$ terms in a Taylor expansion of the curve around $\epsilon=0$ in any coordinate system. Clearly:

$$
G M \rightarrow \infty M \rightarrow I M \rightarrow J M \rightarrow P_{0} M \quad \text { for } I>J
$$

Thus the jets focus on information closer to $\epsilon=0$ than even the germs.

If $x^{a}$ for $1 \leq a \leq N$ are coordinates on $M \approx P_{0} M \approx 0 M$, then we may introduce coordinates $\left\{x_{0}^{a}, x_{1}^{a}, \ldots, x_{J}^{a}\right\}$ for $0 \leq J \leq \infty$ on $J M$ to represent the equivalence class of the curve:

$$
x_{0}^{a}+\epsilon x_{1}^{a}+\frac{\epsilon^{2}}{2 !} x_{2}^{a}+\ldots+\frac{\epsilon^{J}}{J !} x_{J}^{a}
$$

in $I \times M$ (near $\epsilon=0$ this won't leave the chart on which the $x^{a}$ are defined).

The claim here is that $J M$ represents geometrically the perturbation quantities $x_{0}, \ldots, x_{J}$. It may seem strange to go through the infinite dimensional space $P_{1} M$ to get to it, but we shall see (especially when looking at the Hamiltonian structure) that it organizes and simplifies the structures of interest. It is a completely intrinsic and ratural (category theorists would say functorial) operation to go from the original dynamical manifold $M$ to the path space $P_{1} M$ to the jet space $J M$. We shall now show that the dyanamics on $M$ also induces natural dynamics on $P_{1} M$ and then projects from there down to $J M$ where it is the perturbation dynamics we are interested in. Later we will show that a Hamiltonian structure on $M$ leads to Hamiltonian structures on $P_{1} M$ and $J M$. The dynamics $\dot{x}=X(\epsilon, x)$ takes elements of $P_{1} M$ to other elements of $P_{1} M$ and in fact takes equivalence ciasses to equivalence classes for each of $P_{a} M, G M, \infty M, J M$, and $M$. This is what allows us to obtain an induced dynamics on each of these spaces. To determine this dynamics explicitly, we must understand what a tangent vector on each space is.

Intuitively, a vector represents a little perturbation to a point. We define it precisely as an equivalence class of tangent curves, where the curve represents the direction of perturbation and the equivalence class ensures that only the first order motion is reflected in the tangent vector. A point in the path space $P_{1} M$ is a path in $I \times M$. A small perturbation thus gives a nearby path. Each point of the path is perturbed a little bit and we are interested in the first order perturbation. Thus we expect a tangent vector to a point in path space to be a vector feld in $I \times M$ along the corresponding path. A curve $p(\gamma)$ in $P_{1} M$ parameterized by $\gamma$ defines a curve $p(\epsilon, \gamma)$ for each $\epsilon$ through $p(\epsilon, \gamma=0)$ in $I \times M$. The equivalence class of curves in $P_{1} M$ defining a vector thus reduces to an equivalence class of curves in $M$ for each $\epsilon$. We may therefore identify a tangent vector to $p$ in $P_{1} M$ with a field of vectors over $p$ in $I \times M$ such that each vector has no $\frac{\partial}{\partial \epsilon}$ component. For $p \in P_{1} M$ a vector $\tilde{V} \in T_{p}\left(P_{1} M\right)$ is a map:

$$
\bar{V}: I \rightarrow I \times T M
$$

taking $\tilde{V}: \epsilon \mapsto(\epsilon, V(\epsilon))$ where $V(\epsilon) \in T_{p(\epsilon)} M$.

The tangent spaces to the quotient spaces are defined by taking the derivatives of the natural projections. Because $P_{0} M \approx M$, we see that $T P_{0} M \approx T M$. Because $1 M \approx T M$ we see that 
$T 1 M \approx T T M$. Thus the first order perturbation space $1 M$ is naturally $T M$ and the dynamics is a vector field on $T M$ as we saw earlier.

As with all tangent bundles, $T J M$ has a natural coordinate chart, derived from the coordinates $\left\{x_{0}^{a}, \ldots, x_{J}^{a}\right\}, 1 \leq a \leq N$ on $J M$ defined earlier. We obtain coordinates $\left\{x_{0}^{a}, \ldots, x_{J}^{a}\right.$, $\left.v_{0}^{a}, \ldots, v_{J}^{a}\right\}$ by writing the corresponding vector as:

$$
\sum_{a=1}^{N} v_{0}^{a} \frac{\partial}{\partial x_{0}^{a}}+\ldots+v_{J}^{a} \frac{\partial}{\partial x_{J}^{a}} .
$$

We would like to know to which set of components $\left\{v_{0}^{a}, \ldots, v_{f}^{a}\right\}$, the equivalence class of a vector $V(\epsilon)$ on $P_{1} M$ corresponds.

To the path $x^{a}(\epsilon)$ representing a point in $P_{1} M$ corresponds the point coordinatized by:

$$
x_{k}^{a}=\left.\frac{\partial^{k}}{\partial \epsilon^{k}}\right|_{\epsilon=0} x^{a}(\epsilon), \quad 1 \leq a \leq N, \quad 0 \leq k \leq J
$$

in $J M$. To the curve of paths $x^{a}(\varepsilon, \gamma)$ in $P_{1} M$ corresponds the curve:

$$
x_{k}^{a}(\gamma)=\left.\frac{\partial^{k}}{\partial \epsilon^{k}}\right|_{\epsilon=0} x^{a}(\epsilon, \gamma), \quad 1 \leq a \leq N, \quad 0 \leq k \leq J
$$

in $J M$. The vector tangent to this curve in $T P_{1} M$ has coordinates:

$$
V^{a}(\epsilon)=\left.\frac{\partial}{\partial \gamma}\right|_{\gamma=0} x^{a}(\epsilon, \gamma) .
$$

In $T J M$ this corresponds to:

$$
\begin{aligned}
v_{k}^{a} & =\left.\frac{\partial}{\partial \gamma}\right|_{\gamma=0}\left(\left.\frac{\partial^{k}}{\partial \epsilon^{k}}\right|_{\epsilon=0} x^{a}(\epsilon, \gamma)\right) \\
& =\left.\frac{\partial^{k}}{\partial \epsilon^{k}}\right|_{\epsilon=0}\left(\left.\frac{\partial}{\partial \gamma}\right|_{\gamma=0} x^{a}(\epsilon, \gamma)\right) \\
& =\left.\frac{\partial^{k}}{\partial \epsilon^{k}}\right|_{\epsilon=0} V^{a}(\epsilon) .
\end{aligned}
$$

Let us now consider the effect of the dynamics $\dot{x}=X(\epsilon, x)$ on paths. This lifts to a vector field on $P_{1} M$ given by:

$$
\tilde{X} \quad \text { where } \bar{X}(p): \epsilon \mapsto X(\epsilon, p(\epsilon)) .
$$

This is the path space dynamical vector field. In coordinates, the corresponding vector field on $J P$ is:

$$
\begin{gathered}
V_{k}^{a}\left(x_{0}, \ldots, x_{J}\right)=\left.\frac{\partial^{k}}{\partial \epsilon^{k}}\right|_{\epsilon=0} X^{a}\left(\epsilon, x_{0}+\epsilon x_{1}+\ldots+\frac{\epsilon^{J}}{J !} x_{J}\right) \\
=\left.\frac{\partial^{k}}{\partial \epsilon^{k}}\right|_{\epsilon=0} X^{a}\left(\epsilon, x_{0}\right)+\left.k \frac{\partial^{k-1}}{\partial \epsilon^{k-1}}\right|_{\epsilon=0} \sum_{m=1}^{2 N} \frac{\partial}{\partial x_{0}^{m}} X^{a}\left(\epsilon, x_{0}\right) x_{m}^{a}+\ldots
\end{gathered}
$$


which is exactly the perturbation dynamics up to order $J$ obtained in equations (4)!

We have thus found the natural geometric setting for $J$ th order perturbation theory in a certain jet bundle. The picture of the dynamics of paths in $I \times M$ is an extremely fruitful one. One can prove that the solution of the perturbation equations (4) really is the asymptotic expansion of the true solution just by noting that they are the equations of evolution of the jets of the paths evolving under the true dynamics. The coordinates in which the dynamics are expressed are irrelevant as regards the perturbation dynamics and therefore we can do perturbation theory on manifolds and in infinte dimensions as is required for many physical systems. Next we will review modern Hamiltonian mechanics and then show that the perturbation dynamics is Hamiltonian in a natural way if the unperturbed dynamics is.

\section{IV.Geometric Hamiltonian Mechanics}

The evolution of mechanical systems is traditionally described in terms of generalized coordinates $q_{i}$ and their conjugate momenta $p_{i}$. One introduces the Hamiltonian function:

$$
H\left(q_{1}, \ldots, q_{n}, p_{1}, \ldots, p_{n}\right)
$$

and the Poisson bracket:

$$
\{f, g\}=\sum_{i=1}^{n}\left(\frac{\partial f}{\partial q_{i}} \frac{\partial g}{\partial p_{i}}-\frac{\partial f}{\partial p_{i}} \frac{\partial g}{\partial q_{i}}\right)
$$

of two functions of $q_{i}$ and $p_{i}$. Any observable $f$ evolves according to the evolution equation:

$$
\dot{f}=\{f, H\} \text {. }
$$

For a detailed description of the modern approach see references [7], [8], and [30]. The modern perspective regards the particular coordinates $p_{i}$ and $q_{i}$ as physically irrelevant. Just as general relativity isolates the physically relevant essence of local coordinates in a metric tensor, modern classical mechanics views the Poisson bracket structure (not necessarily expressed in any coordinate system) as the physical entity. Just as physics in spacetime is invariant under transformations that preserve the metric, physics in phase space is invariant under the canonical transiormations which preserve the Poisson bracket. In the modern viewpoint one proceeds axiomatically and does not require c anonical coordinates. Dynamics occurs on a Poisson manifold. This is a manifold of states with a Poisson bracket defined on it. From this viewpoint a Poisson bracket is a bilirear map from pairs of functions to functions which makes the space of functions into a Lie algebra and acts on products like a derivative does:

$$
\begin{array}{ll}
\text { I. Bilinearity: } & \left\{a f_{1}+b f_{2}, g\right\}=a\left\{f_{1}, g\right\}+b\left\{f_{2}, g\right\} \\
\text { 11. Anti-symmetry: } & \{f, g\}=-\{g, f\} \\
\text { III. Jacobi's identity: } & \{f,\{g, h\}\}+\{g,\{h, f\}\}+\{h,\{f, g\}\}=0 \\
\text { IV. Derivation property: } & \{f, g h\}=\{f, g\} h+\{f, h\} g .
\end{array}
$$


The Haniltonian is a function on the Poisson manifold. The evolution of local coordinates $z^{i}$ is obtained from a Hamiltonian $H$ and the Poisson bracket $\{$,$\} via:$

$$
\dot{z}^{i}=X_{H} \cdot z^{i}=\left\{z^{i}, H\right\}
$$

$X_{H}$ is the Hamiltonian vector field associated with $H$ and defines a dynamical system. The fourth property of a Poisson bracket implies the useful expression:

$$
\{f, g\}=\sum_{i, j} \frac{\partial f}{\partial z^{i}}\left\{z^{i}, z^{j}\right\} \frac{\partial g}{\partial z^{j}} .
$$

Thus the Poisson bracket is equivalent to an antisymmetric contravariant two-tensor:

$$
J^{i j} \equiv\left\{z^{i}, z^{j}\right\}
$$

If this is nondegenerate, its inverse $\omega \equiv J^{-1}$ is a closed, nondegenerate two-form called a symplectic structure. In this case our Poisson manifold is known as a symplectic manifold. The terminology is due to Herman Weyl. If one works in canonical coordinates, the matrix $w$ has a square equal to minus the identity matrix. In this sense, $w$ was thought of as a complex structure. Intrinsically, however, $\omega$ is a two-form and thus takes a vector and returns a one-form, preventing us from squaring $\omega$. One needs a metric to "lower an index" and obtain a complex structure. To eliminate this confusion, Weyl took the Latin roots com and plex and converted them to their Greek equivalents sym and plectic.

Because we do not require nondegeneracy, a Poisson manifold is a more general notion than a symplectic manifold. If $J$ is degenerate, then there are directions in phase space in which no Hamiltonian vector field can point. The available directions lie tangent to submanifolds which fill out the Poisson manifold and on which $J$ is nondegenerate. The highest dimensional of these form a foliation of their union and so are known as symplectic leaves. The only usage of the term symplectic in English is to describe a small bone in the head of a fish. Because Poisson is French for fish, the lower dimensional symplectic submanifolds are known as symplectic bones (the terminology is due to Alan Weinstein) ${ }^{29}$. Together the symplectic leaves and the symplectic bones fill out the Poisson manifold and any Hamiltonian dynamics is restricted to lie on a single bone or leaf. Any function which is constant on each bone and leaf Poisson commutes with every other function. Any function which Poisson commutes with every function is automaically a constant of the motion, regardless of the Hamiltonian and is called a Casimir function.

A natural symplectic manifold arises from Lagrangian mechanical systems on a configuration space $C$. The Lagrangian $L$ lives on the tangent bundle $T C$ (velocities being tangent to the curves of motion in configuration space are naturally tangent vectors). Hamiltonian mechanics takes place on the cotangent bundle $T^{\bullet} C$ (momenta, being derivatives of $L$ with respect to velocity, are naturally dual to velocities and thus are covectors). $T^{*} C$ has a natural symplectic structure $\omega=-d \theta$ where $\theta$ is an intrinsically defined one-form. It acts on tangent vectors $v$ to $T^{*} C$ at the 
point $(x, \alpha)$ by first pushing them down to the base $C$ by the natural projection $\pi$ which gives the basepoint of a covect $r$ and then inserting the result into the one-fors $\alpha$ on $C$. Thus:

$$
\theta(v)=\alpha(\pi \cdot v)
$$

In coordinates $q^{a}$ on $C, \theta=p_{a} d q^{a}$ and $\omega=d q^{a} \wedge d p_{a}$. This generalizes the usual structure in terms of canonical $p$ 's and $q$ 's to configuration spaces which are manifolds. Symmetry is responsible for most of the simplified systems about which we perturb and plays as intimate role in our geometric iheory. We therefore introduce some key modern ideas and basic examples relating to Hamiltonian symmetry.

\section{Hamiltonian Systems with Symmetry}

Perhaps the central advantageous feature of systems with a Hamiitonian structure is a generalization of Noether's theorem relating symmetries to conserved quantities. Ncether considered symmetries of the Lagrangian under variations of configuration space. One may introduce generalized coordinates $q_{1}, \ldots, q_{n}$ where $q_{2}, \ldots, q_{n}$ are constant under the symmetry transformation and $q_{1}$ varies with the transformation. For example we might take the configuration space to be ordinary Euclidean 3-space where the action of the symmetry is translation in the $x$ direction, and utilize the coordinates $q_{1}=x, q_{2}=y, q_{3}=z$. That $L$ is invariant means that it doesn't depend on $q_{1}$, i.t. $q_{1}$ is an ignorable coordinate. The Euler-Lagrange equations:

$$
\frac{d}{d t}\left(\frac{\partial L}{\partial \dot{q}}\right)-\frac{\partial L}{\partial q}=0
$$

show that in this case the momentum $p_{1}=\frac{\partial L}{\partial \dot{q}_{1}}$ conjugate to $q_{1}$ is actually a constant of the motion.

By going to a Iamiltonian description in terms of Poisson bräckets we may extend Noether's theorem in a fundamcutal way. We may consider a one-parameter symmetry transformation of the whole phase space as opposed to just configuration space. If this transformation preserves the Hamiltonian and the Poisson bracket (i.e. is a canonical transformation) then it is associated with a conserved quantity. We will see that this extension of Noether's theorem is essential in the case of gyromotion and in other examples.

One-parameter families of canonical transformations of this type may be represented as the "time" $s$ evolution of some function $J$, treated snomentarily as a Hamiltonian. Parameterizing our transformation by $s$ and labelling points in phase space by $\tilde{z}$, the solution $\underline{z}(s)$ of

$$
\frac{d \underline{z}}{d s}=\{\underline{z}, J\} \quad \underline{z}(s=0)=\underline{s}_{0}
$$

is the canonical transformation generated by $J$. 
If the transformation generated by $J$ is a symmetry of $H$ then:

$$
\begin{aligned}
\frac{d H}{d s}=0 & =\sum_{i} \frac{\partial H}{\partial z^{i}} \frac{d z^{i}}{d s} \\
& =\sum_{i} \frac{\partial H}{\partial z^{i}}\left\{z^{i}, J\right\} \\
& =\{H, J\} \\
& =-\{J, H\} \\
& =-j
\end{aligned}
$$

So $J$ is a conserved quantity.

We now consider the case in which the solutions of:

$$
\frac{d \underline{z}}{d s}=\{\underline{z}, J\}
$$

are all closed curves (every orbit is periodic). We will call these closed orbits loops. The symmetry transformation is then said to be a circle action on phase space.

For example we might consider rotation by $\theta$ in $J, \theta$ space. In this case phase space looks like a cylinder. The Poisson bracket is:

$$
\{f, g\}=\frac{\partial f}{\partial \theta} \frac{\partial g}{\partial J}-\frac{\partial f}{\partial J} \frac{\partial g}{\partial \theta} .
$$

$J$ generates the dynamics:

$$
\begin{aligned}
& \frac{d \theta}{d s}=\{\theta, J\}=1 \\
& \frac{d J}{d s}=0
\end{aligned}
$$

which just rotates the cylinder.

In studying the dynamics of a Hamiltonian $H$ symmetric under a circle action generated by $J$, we may make two simplifications which together comprise the process of reduction. This procedure was defined by Marsden and Weinstein ${ }^{32}$ in a more general setting that we will describe shortly. The process unifies many previously known techniques for simplifying specific examples of Hamiltonian systems.

1. Because $J$ is a constant of the motion, the surface $J=$ constant in phase space is left invariant by the dynamics and so vee may restrict attention to it.

2. The symmetry property of $H$ implies that if we take a solution curve $z(t)$ of the equation $\underline{\dot{z}}=\{\underline{z}, H\}$ and let it evolve for a "time" s under the dynamics $\underline{\dot{z}}=\{\underline{z}, J\}$ then we obtain another solution curve of $\underline{\underline{z}}=\{\underline{z}, H\}$. In fact the dynamics of $H$ takes an entire loop into other entire loops.

The dynamics around loops is easy to solve for:

$$
\dot{\theta}=\frac{\partial H}{\partial J} .
$$


Notice that $\theta$ is not uniquely defined but $\dot{\theta}$ is. We are interested in the problem of finding the dynamics from loop to loop. We want to project the original dynamics on phase space $P$ down to a space $P / S^{1}$ whose points represent whole loops in $P$. Let us call $P / S^{1}$, the space of loops and $\pi: P \rightarrow P / S^{1}$, the projection mapping loops in $P$ to points in $P / S^{1}$. For example, when $P=J, \theta$ space the prcjection mapping takes $J, \theta$ to $J$. Thus the second simplification is to consider dynamics on the space of loops $P / S^{1}$.

Performing botb of these operations-restricting to $J=$ constant and considering the space of loops- leaves us with a space:

$$
R \equiv P /\left.S^{1}\right|_{J=\text { constant }}
$$

with two dimensions less than $\boldsymbol{P}$ called the reduced space.

We have seen that the dynamics on $P$ naturally determines dynamics on $R$. The key importance of $R$ is that $R$ 's dynamics is itself Hamiltonian. For this statement to make sense we need to find a Hamiltonian and a Foisson bracket on $\mathrm{R}$. These are the so called reduced Hamiltonian and reduced Poisson bracket.

The original Hamiltonian $H$ on $P$ is constant on loops by the symmetry condition. We may take the value of the reduced Hamiltenian at a point of $R$ to be the value of $H$ on the corresponding loop in $P$.

To take the reduced Poisson bracket of two functions $f$ and $g$ on $R$, we consider any two functions $\hat{f}$ and $\hat{g}$ on $P$ which are constant on loops and agree with $f$ and $g$ when restricted to $J=$ constant and projected by $\pi$ to $R$. The Poisson bracket on $P$ of $\hat{f}$ and $\hat{g}$ will be constant on loops and its value on $J=$ constant will be independent of how $\hat{f}$ and $\hat{g}$ were extended as functions on $J$ (because they are constant on loops: $\{\hat{f}, J\}=0$ and $\{\hat{g}, J\}=0$ so $\{\hat{f}, \hat{g}\}$ is independent of $\partial \hat{f} / \partial J$ and $\partial \hat{g} / \partial J$ ). Thus the value of the reduced Poisson bracket or $R$ of $f$ and $g$ is the value on the corresponding loop in $P$ of the Poisson bracket of any two extensions $\hat{f}, \hat{g}$ that are constant on loops.

In examples we often introduce a coordinate $\theta$ describing the position on a loop. We may then treat $P / S^{1}$ as the set $\theta=0$ (at least locally). In this case $R$ is the subset $\theta=$ constant, $J=$ constant of $P$. The reduced Hamiltonian on $R$ is just the value of $H$ on this subset of $P$. To calculate the value of the Poisson bracket of two functions on $P$ on this surface, we need only cheir first derivatives there.

If the iunctions are constant on loopg (i.e. independent of $\theta$ ), then the derivative $\partial / \partial \theta$ is zero. The dependence on $J$ is irrelevant, so we may take the derivative $\partial / \partial J$ to be zero. Plugging these two expressions into the Poisson bracket on $P$ gives us the exprission for the reduced Poisson bracket on $R$.

\section{Example: Centrifigal Force}

We consider a particle on a two-dimensional planc moving in a rotationally symmetric potential. The phase space is then $T^{\bullet} \mathfrak{R}^{2}$ with coordinates $x, y, p_{x}, p_{y}$. The Poisson bracket is the 
canonical one:

$$
\{f, g\}=\frac{\partial f}{\partial x} \frac{\partial g}{\partial p_{x}}-\frac{\partial f}{\partial p_{x}} \frac{\partial g}{\partial x}+\frac{\partial f}{\partial y} \frac{\partial g}{\partial p_{y}}-\frac{\partial f}{\partial p_{y}} \frac{\partial g}{\partial y} .
$$

The Hamiltonian is taken to be:

$$
H=\frac{1}{2 m}\left(p_{x}^{2}+p_{y}^{2}\right)+V\left(\sqrt{x^{2}+y^{2}}\right) .
$$

The symmetry on phase space is given by the evolution of the equations:

$$
\begin{array}{ll}
\frac{d x}{d s}=-y & \frac{d y}{d s}=x \\
\frac{d p_{x}}{d s}=-p_{y} & \frac{d p_{y}}{d s}=p_{x}
\end{array} .
$$

We may think of a point in phase space as a point in the plane $(x, y)$ with a vector attached $\left(p_{x}, p_{y}\right)$. The action of the symmetry is to rotate the plane about the origin, vector and all.

The Hamiltonian depends only on the radial distance and the magnitudie of the momentum vector and so is ciearly left invariant by this rotation. The rotation is a canonical transformation with generator $J$ satisfying:

$$
\frac{d f}{d s}=\{f, J\}=x \frac{\partial f}{\partial y}-y \frac{\partial f}{\partial x}+p_{x} \frac{\partial f}{\partial p_{y}}-p_{y} \frac{\partial f}{\partial p_{x}}
$$

for any $f$. Taking $f=x, y, p_{x}, p_{y}$ gives:

$$
\frac{\partial J}{\partial p_{x}}=-y \quad \frac{\partial J}{\partial p_{y}}=x \quad \frac{\partial J}{\partial x}=p_{y} \quad \frac{\partial J}{\partial y}=-p_{x} .
$$

Thus we see that the generator is $J=x p_{y}-y p_{x}$, i.e. the angular momentum. We may label a loop by the value of $x, p_{x}$, and $p_{y}$ when $y=0$ and $x \geq 0$. $J$ on this subset ig just $x p_{y}$. These then form coordinates on the space of loops $P / S^{1}$.

To get the reduced space we set $J$ to the constant value $\mu$. Thus we may take the coordinates on $P /\left.S^{1}\right|_{J=\mu}$ to be $x$ and $p_{x}$ when $y=0$ and $p_{y}=\mu / x$. On $R$ we have:

$$
\frac{\partial}{\partial \theta}=x \frac{\partial}{\partial y}+p_{x} \frac{\partial}{\partial p_{y}}-\frac{\mu}{x} \frac{\partial}{\partial p_{x}} .
$$

Setting this to zern gives the reduced bracket by plugging

$$
\frac{\partial}{\partial y}=\frac{\mu}{x^{2}} \frac{\partial}{\partial p_{x}} \quad \text { and } \quad \frac{\partial}{\partial p_{y}}=0
$$

into the expression for $\{f, g\}$ :

$$
\{f, g\}=\frac{\partial f}{\partial x} \frac{\partial g}{\partial p_{x}}-\frac{\partial f}{\partial p_{x}} \frac{\partial g}{\partial x} .
$$

The reduced bracket in this case is just the canonical bracket on $x, p_{x}$ space.

The reduced Hamiltonian is obtained by restricting the original Hamiltonian to our subset and is given by:

$$
H=\frac{p_{z}^{2}}{2 m}+\left(\frac{\mu^{2}}{2 m} \frac{1}{x^{2}}+V(x)\right)
$$


Note the effective potential due to reduction, that represents the centrifugal force.

\section{Higher Dimensional Symmetries 32}

Quite often physical systems are blessed with more than one dimension of symmetry. In keeping with the philosophy of not making unphysical choices it is natural to consider the process of reduction in the presence of an arbitrary Lie group of symmetry. A Lie group is a group which is also a manifold, such that the group operations respect the smoothness structure. A Hamiltonian system with symmetry consists of a Poisson manifold $M$, a Hamiltonian $H$, and a group $G$ that acts on $M$ so as to preserve both $H$ and the Poisson bracket $\{$,$\} . The tangent space$ of $G$ at its identity may be identified with the Lie algebra $g$ of the group and represents group elements infinitesimally close to the identity. The action of an infinitesimal element of $G$ on $M$ perturbs each point of $M$ by an infinitesimal amount. Thus each element $v$ of the Lie Algebra of $G$ naturally determines a vector field on $M$. The action of the one-dimensional subgroup to which $v$ is tangent on $M$ is given by the flow of this vector field. That the group action preserves the Poisson bracket, implies that this vector field is actually Hamiltonian. Thus we may associate to $v$ a Hamiltonian funtion which generates this vector field (at least locally). If $G$ is $n$ dimensional, and we pick a basis for $g$, then the group action gives us $n$ corresponding Hamiltonian functions on $M$. So as not to prefer one basis over another, we collect these $n$ numbers at each point of $M$ into $z$ vector. This vector pairs naturally with an element of $g$ (to give the value of the function which generates the action of that element) and so the collection of $n$ Hamiltonians is a vector in the dual of the Lie algebra $g^{*}$ at each point of $M$. Thus with every Hamiltonian group action of $G$ on $M$, there is a natural map calied the momentum map from $M$ to $g^{*}$ which collects together the generators of the infinitesimal action of $G$ on $M$. For a mechanical system in $R^{3}$ which is translation invariant, the momentum map associates with each point in phase space the total linear momentum of the system in that state. If the Hamiltonian is rotationally symmetric, then the momentum map gives the total angular momentum in each state (thus we see that angular mornentum isn't naturally a vector in $R^{3}$, rather it takes its values in the dual of the Lie algebra of the rotation group $\left.s o(3)^{*}\right)$. When we talked about reduction in the one dimensional case above, the generatur of the action $J$ was the momentum map.

Does reduction work for higher dimensional symmetries? If the group is commutative, then we may apply the one-dimensional proceedure repeatedly to elininate two degrees of freedom for each dimension of symmetry. If we are able to eliminate all dimensions of phase space in this way, then the system is integrable. If orbits are bounded then the group is a toris in this case. Locally we may define angle variables on the toroidal group orbits and the corresponding action variables form the momentum map. Recall that there were two steps in the reduction of systems with one dimension of symmetry, each of which eliminated one dimension of the phase space and that either could be performed first. One was to restrict to a level set of the generating function and the other was to drop down to the orbit space (space of loops). For non-conmutative groups, we may again perform either of these two operations, but each gets in the way of subsequently 


\section{Some Natural Group Actions:}

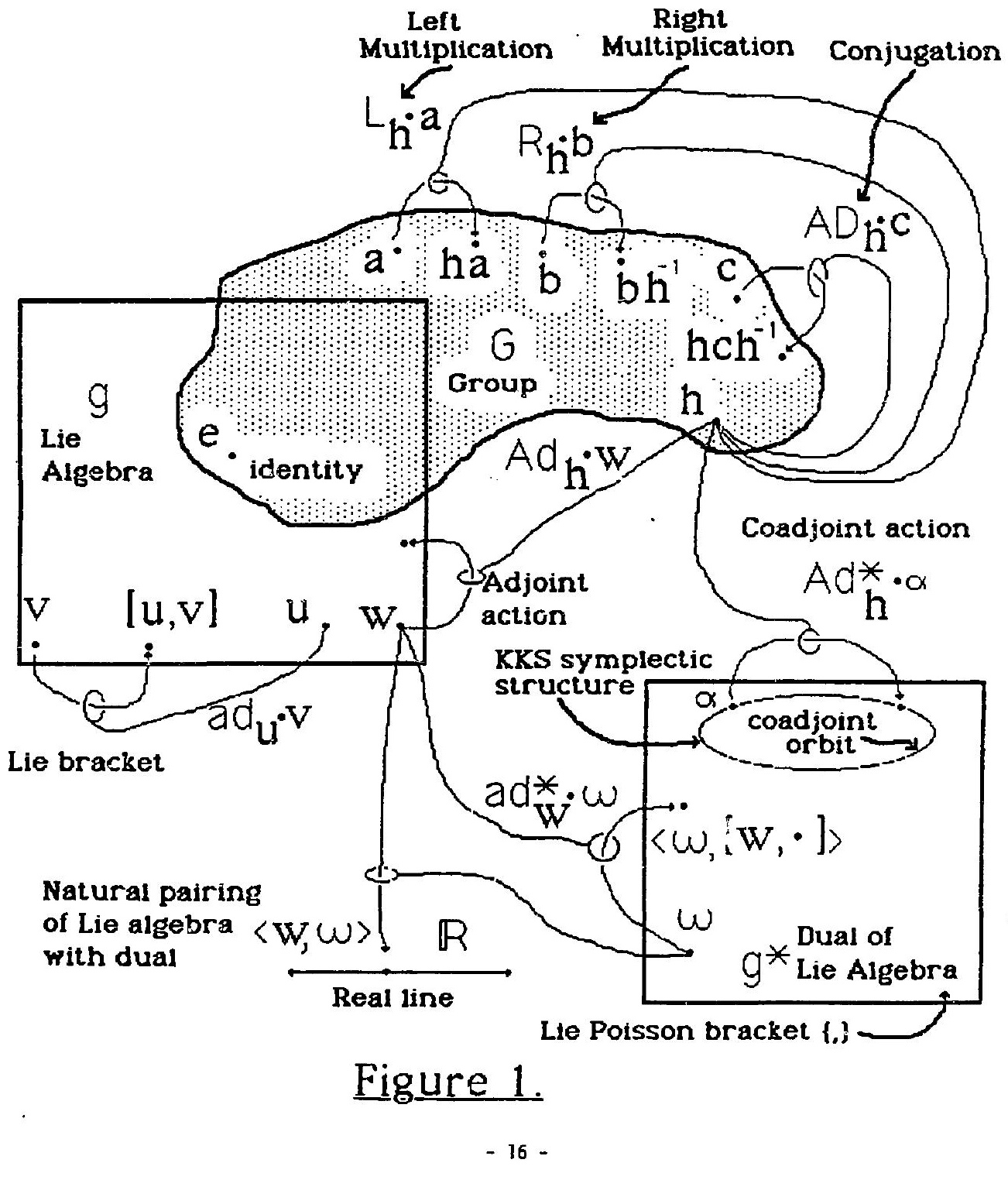


performing the other. The main issue here is that while the Hamiltonian is invariant under the group action, the momentum map is not. Consider the example of a mechanical system in a spherically symmetric potential so that the rotation group acts on phase space as a symmetry and the momentum map is the total angular momentum. While the energy is left unchanged as we rotate the state, the angular momentum is rotated just like a vector in $R^{3}$. This action of $S O(3)$ on 2 ' 3 dual of its Lie algebra is known as the coadjoint action.

Let us digress a bit on the structure of Lie groups to make this point clearer. As shown in the diagram in figure 1, every Lie group has three natural actions on itself. If $h$ is an element of $G$, then we may multiply on the left by $h$ to get the action $L_{h}$, we may multiply on the right by $h^{-1}$ (inverse so that $R_{f h}=R_{f} F_{h}$ ) to get $R_{h}$, and conjugate by $h$ (i.e. $c \mapsto h c h^{-1}$ ) to get the action $A D_{h}$. Conjugation captures the noncommutativity of the group that is at issue here. $A D_{h}$. leaves the identity invariant (since $h \cdot e \cdot h^{-1}=e$ ). We may therefore take the derivative of $A D_{h}$. at the identity to get a linear map from the Lie algebra to itself denoted $A d_{h} \cdot$. Ad is actually a representation of $G$ on its Lie algebra sometimes called the fundamental representation. If sve take the derivative of $A d_{h}$. in the $h$ variable we get an action ad of the Lie algebra on itself. The action of an element $u \in g$ is none other than Lie bracket with $u$, so $a d_{u} \cdot v=[u, v]$. We have seen that the dual of the Lie algebra $g^{*}$ plays an important role in Hamiltonian symmetries. Any time you have a linear transformation $L$ acting on a vector space $V$, you can define its adjoint $L^{*}$ acting on $V^{*}$ by requiring that $\left\langle L^{*} a, v\right\rangle=\langle a, L v\rangle$. The adjoir $t$ of $A d_{h}$. is called the coadjoint action of $G$ on the dval of its Lie algebra $g^{*}$ and is written $A d_{h}^{*}$. The action of the rotation group on angular momenta that we discovered above is an example of this. One usually sequires that a momentum mapping be equivariant as in this example. This means that the value of the momentum map varies as the group acts on the phase space according to the coadoint action: $J(g \cdot x)=A d_{g}^{*} \cdot J(x)$.

Let us now try to mimic the reduction procedure in this noncommutative case. First we restrict attention to the subset of phase space $J=\mu$, a constant. The dynamics restricts to this subset because $J$ is a constant of the motion. The whole group $G$ does not act on this subset however, because a general element of $G$ will change the value of $J$. The subgroup of $G$ which leaves $\mu$ invariant under the coadjoint action (known as $\mu$ 's isotropy subgroup $G_{\mu}$ ) will act on this subset and we may drop the dynamics down to its orbit space. The resulting space: $\left.M\right|_{J=\mu} / G_{\mu}$ has a natural symplectic structure and the Hamiltonian restricted to it generates the projected dynamics. For the rotation group example, we restrict to states with a given total angular momentum (eliminating 3 dimensions) and then forget about the angle of rotation about the axis defined by that angular momentum(eliminating one more). The result is a phase space of four dimensions lower than we started with.

We may obtain the same result in another way. Consider the orbit of a particular element $\mu$ of the dual of the Lie algebra under the coadjoint action. This coadjoint orbit $O_{\mu}$ has a natural symplectic structure we will discuss moinentarily. For the rotation group the coadjoint orbits are spheres of constant total angular momentum (and the origin). The orbit space of $M$ modulo $G$ has a natural Poisson structure (the bracket of $G$ invariant functions is $G$ invariant) which 
is not typically symplectic. The symplectic leaves project onto the coadjoint orbits under the momentum map. The inverse image of a whole coadjoint orbit under the momentum map is left invariant under the group action on $M$ : The orbit space $\left.M\right|_{J-1 O_{\mu}} / G$ is the same reduced space we constructed above. For the rotation group this consists of restricting to states with a given total magnitude of angular momentum and then modding out by the whole rotation group.

An inportant example of reduction applies to mechanical systems whose configuration space is the symmetry group itself. We will see that the free rigid body and the perfect fluid are examples of this type, a fact first realized by Arnold ${ }^{33}$. The phase space $M$ is then $T^{*} G$ and the $G$ action is the canonical lift to $T^{*} G$ of left or right multiplication. The $G$ orbits have one point in each fiber and so we may identify the orbit space with the cotangent space at the identity, i.e. the dual of the Lie algebra. The momentum map is then the identity and the coadjcint orbits receive a natural symplectic structure, being the reduced spaces. These symplectic structures are known as Kirilloy- Kostant-Souriou (KKS) symplectic structures. If we just consider the orbit space $T^{*} G / G$ then we obtain a natural Poisson bracket on $g^{*}$ already known to Sophus Lie (and so called the Lie-Poisson bracket). Explicitly it is:

$$
\{f, g\}(\alpha)=\left\langle\alpha,\left[\frac{\delta f}{\delta \alpha}, \frac{\delta g}{\delta \alpha}\right]\right\rangle
$$

where $\alpha \in g^{*}, f$ and $g$ are functions on $g^{*},[$,$] is the Lie algebra bracket, and <,>$ is the natural pairing of $g$ and $g^{*}$. This bracket is behind many of the nontrivial Poisson structures recently discovered in various areas of physics.

To specify the configuration of a free rigid body, we give some reference configuration and every other configuration is uniquely specified by giving the element of the rotation group that acts on the reference to give the desired one. Thus the configuration space is identifiable with the group $S O(3)$ itself. The state including angular velc city is naturally a point in $T S O(3)$ and the state including angular momentum is a point in $T^{*} S O(3)$. A priori, there is no way of comparing the angular velocity or momentum in one configuration with that in another. Using the group action, however, we may push all velocities to velocities at the identity (i.e. velocities on the reference configuration) which may be identified as elements of the Lie algebra. Both left and right multiplication can bring us to the identity since they act on the group transitively. Consider a path at the identity (for example a rotation about the $z$ axis) to which a given element of $g$ is tangent. Left multiplication by $h \in G$ means move along the path and then rotate by $h$. Thus the path is associated with the body and we get the angular velocity in the body-fixed frame. Multiplying on the right means rotate first by $h$, then tollow the path. The path applied is independent of the configuration of the body (described by $h$ ) and so its tangent represents angular velocity in the space-fixed frome. Similarly, left multiplication gives angular momentum in the body-fixed frame and right multiplication gives it in the space-fixed frame. At a configuration represented by $h \in G$, the map from $g$ to $g$ that takes spatial angular velocity to body angular velocity is the adjoint action of $h$. Similarly, the map from $g^{*}$ to $g^{*}$ that takes spatial angular momentum to body angular momentum is the coadjoint action. The energy only depends on the angular momentum in the body (the orientation in space is irrelevant for a free rigid body) 
and so the Hamiltonian on $T^{*} S O(3)$ is invariant under the cotangent lift of left multiplication and we are indeed in the situation described above. If we drop down to the orbit space of this left multiplication, we get a Poisson bracket and Hamilionian on the three dimensional space of angular momenta in the body. The dynamics on this space is exactly Euler's equations. The Poisson bracket is explicitly given by:

$$
\left\{J_{x}, J_{y}\right\}=J_{x}
$$

plus cylic permutations. The total angular momentum $J_{x}^{2}+J_{y}^{2}+J_{z}^{2}$ is a Casimir function and so is automatically conserved. The coadjoint orbits (and so the symplectic leaves and bones) are the spheres of constant total angular momentum and the origin. The area element on the spheres is the two-form which is the KKS symplectic strucuture.

In an exactly analogous way, we may consider the Hamiltonian structure of a perfect Huid. If we choose a reference configuration, then to get any other configuration we apply a unique diffeomorphism (volume preserving if the fluid is incompressible). Thus the configuration space may be identified with the group of diffeormophisms of the region in which the fluid resides. The state of the fluid plus its velocity field is represented by a point in the tangent bundle of the group. The phase space gives the state of the fluid plus the momentum density and so is the cotangent bundle of the group. Again we may identify velocities and momenta with elements of the Lie algebra and its dual by left or right multiplication. Right multiplication gives the Eulerian velocity or momentum feld in space. Left multiplication gives them for material points in the reference configuration. Here, in contrast to the rigid body case, the energy depends only on the spatial momentum (which fluid particle is where is irrelevant) and so the Hamiltonian is right invariant. Dropping to the orbit space gives us dynamics for the spatial momentum density, i.e. Euler's fluid equations, in Hamiltonian form.

For gases and plasmas, the state of the system is represented by the particle distribution function on single-particle phase space. This distribution function evolves by the action of symplectomorphisms (i.e. canonical transformations) of this phase space. The group of symplectomorphisms has the Hamiltonian vector fields as its Lie algebra. We may identify this with the space of functions on the phase space with the Lie bra 'ret being the Poisson bracket of functions. The dual of the Lie algebra is then densities on phase space, which we may use to describe the kinetic state of plasmas and gases. The coadjoint action just pushes the density around by the symplectomorphism. One coadjoint orbit comes from considering a delta distribution on phase space. The symplectomorphisms push it all over phase space to give a coadjoint orbit that is identifiable with the original phase space. In fact the KKS symplectic structure is exactly the original symplectic structure. This shows that every sy mplectic manifold is a coadjoint orbit. A delta distribution whose support is a loop or torus in phase space shows that the space of loops with a given action (or tori with given actions around their fundamental loops) form a symplectic manifold. 
Let us now relate this geometric Hamiltonian mechanics to the geometric perturbation theory we discussed earlier. We will see that the $J$ th order perturbed dynamics has a natural Hamiltonian structure if the exact dynamics does.

The first thing to note is that the path space dynamics is Hamiltonian. This is not surprising if we think of the path space as a kind of direct integral of the phase spaces at each $\epsilon$. The dynamics at different $\epsilon$ 's are completely independent (except for the fact that the paths are smooth). If we had the product of only two Hamiltonian systems (instead of a continuum of them) then we would get the correct dynamics from a symplectic structure which is the sum of the pullback to the product of the individual symplectic structures and a Hamiltonian which is the sum of the pulled back Hamiltonians. Extending this construction to a continuum of multiplicands leads to the symplectic structure:

$$
\tilde{\omega}_{p}\left(\tilde{V}_{1}, \tilde{V}_{2}\right) \equiv \int_{0}^{1} \omega_{p(\epsilon)}\left(V_{1}(\epsilon, p(\epsilon)), V_{2}(\epsilon, \Gamma(\epsilon)\}\right) d \epsilon
$$

The analog of the sum of Hamiltonians is:

$$
\tilde{H}(p) \equiv \int_{0}^{1} H(\epsilon, p(\epsilon)) d \epsilon .
$$

The dynamics these two generate is indeed the correct path space dynamics. In the case of a product of a fnite number of Hamiltonian systems, we are actually allowed to take any linear combination of the symplectic structures (instead of a straight sum) as long as no coefficient vanishes and we take the same linear combination of Hamiltonians. If a coefficient vanishes, that factor has no dynamics. For our perturbation dynamics then, we want to ignore the region in the interval that is away from $\epsilon=0$.

In fact if we substitute the $J$ th derivative of a delta function into the integrals in (49) and (50) we get the correct perturbation dynamics on $J M$. If the Poisson bracket on $M$ is $\left\{x^{a}, x^{b}\right\}=J^{a b}$ then the bracket on $J M$ is:

$$
\left\{x_{k}^{a}, x_{m}^{b}\right\}=J^{a b} \frac{k ! m !}{J !} \delta_{k, J-m}
$$

and the Hamiltonian is:

$$
\left.\bar{H}\left(x_{0}, \ldots, x_{J}\right) \equiv \frac{d^{J}}{d \epsilon^{J}}\right|_{\epsilon=0} H\left(\epsilon, x_{0}+\epsilon x_{1}+\ldots+\frac{\epsilon}{J !} x_{J}\right) .
$$

Together these give the correct perturbation dynamics. Notice that the Oth order variables are paired with $J$ th order variables, 1st order with $J$ - 1st order, etc.

From the above coordinate description it is not clear that this bracket is in fact intrinsic. We may show this by considering the iterated tangent bundle to $M$. The tangent bundle to a symplectic manifold has a natural symplectic structure. If $\omega$ is the structure on $M$, then we may use it to identify $T M$ and $T^{*} M, T^{*} M$ has a natural symplectic structure, which we defined in (29). The structure on $T M$ is obtained by pulling $T^{*} M^{\prime}$ 's back using the identification supplied by $\omega$. This operation may be iterated to give symplectic structures on the iterated tangent bundles: 
$T T M, T T T M, T T T T M$, etc. The $J$ th order jets uaturally embed into the $J$ th iterated tangent bundle. If the symplectic structure on $T^{J} M$ is pulled back to $J M$ we obtain the jet Poisson bracket in equation (51).

The symplectic structure on $T M$ may be thought of as the first derivative of the original symplectic structure ${ }^{34}$. The jet bracket may be thought of as the $J$ th derivative. Choose $J$ sheets spaced evenly in $I \times M$. The path dynamics projects down to the product of these sheets. We may map this structure to $J M$ with arbitrary coefficients. ii these coefficients are chosen to give a nonsingular result as the sheet spacing goes tc zero, we again obtain the jet symplectic structure and Hamiltoni . This shows that the perturbation bracket and Hamiltonian are in essence $J$ th derivatives of the path structures.

We have seen that when the Poisson bracket is degenerate, non-degenerate symplectic leaves and bonss are injected into the Poisson manifold as submanifolds. If a closed two-form is desenerate, then we project out the degenerate directions to obtain a symplectic manifold. The fact that the two-form is closed implior sat the annibilated directions satisfy the conditions of Frobenius's theorem and so lie tangent to smo. submanifolds which we may then project along (at least locally). We have used an example of this construction above. If we insert the $J$ th derivative of a delta function into the path symplectic integral (49), we obtain a degenerate, closed two-form on the path space $P_{1} M$. The projection e eliminating the degenerate directions is exactly the projection from path space down to the jet space $J M$. The resultirg symplectic structure is the jet perturbation structure. If we have a Hamiltonian system with an invariant submanifold, we may attempt to obtain the restricted dynamics in Hamiltonian form by pulling back the symplectic structure. The resulting two-form will be closed but may not be non-degenerate. If things are nice globally, we may apply the above projection. A special case of this demoritrates that the jet construction contains as a special case the linearized dynarnics of a Hamiltonian system around a fixed point. We consider the 2-jet space $2 M$. The submanifold of jets with base point equal to the fixed point is an invariant submanifold. Because the zero order base directions are paired with the second order directions in (51), restricting to a given basepoint makes the second order directions degenerate. Projecting these out leaves us with only the first order jets at the fixed point (i.e. the tangent space there). Tese are paired with themselves by the second order bracket according to the original symplectic structure at the fixed point. The second order Hamiltonian (52) gives the quadratic piece of the Taylor expinsion in the $x_{1}$ variables. Together these give the linearized flow in the tangent space of the fixed point as a Hamiltonian system. The situation in Poisson manifolds is more complex ${ }^{29}$. If the fxed point is a symplectic leaf, we take the Poisson bracket at the point, the quadratic part of the Hamiltonian in the leaf direction, and the linear part of the Hamiltonian across leaves. The bones are more difficult.

We have seen how important symmetry and its related concepts are in Hamiltonian mechanics. How do the symmetry operations intermix with the perturbation operations? A Hamiltonian $G$ action on $M$ lifts to both the path space $P M$ (just push the whole path around by the group action) and the jet space $J M$ (just push the jet around). The corresponding momentum maps are just the integral along a path of the $M$ momentum map and the same integral with the $J$ th 
derivative of a delta function thrown in. Both are equivariant.

When considering reduction we quickly see that these groups are not of high enough dimension. A 4 dimensional phase space with a 1 dimensional symmetry drops down to 2 dimensions. The first order perturbation space has 8 dimensions. In the presence of symmetry we expect to be able to drop this down to the first order perturbation space of the 2 dimensional reduced space. The above group action can only eliminate 2 dimensions instead of the needed 4 and so we expect a larger group to act. This is indeed the case. It makes sense to multiply two paths in a group by multiplying pointwise. Thus $P G$ is an infinite dimensional "Lie" group and its "Lie" algebra is the path space of $G$ 's Lie algebra $g$. $P G$ has a Hamiltonian action on the path space $P M$ by multiplying the point $p(\epsilon)$ by the group element $g(\epsilon)$. The momentum map sends a path in $M$ to a path in $g^{*}$ gotten by applying $M^{\prime}$ 's momentum map to each $\epsilon$. In an exactly analogous way, we may define the group $J G$ of $J$-jets of paths in $G$ with Lie slgebra being $J$-jets of paths in: This acts in a Hamiltonian and equivariant way on the perturbation space $J M$. The momentum map is obtained by extending a jet to any consistent path, taking the path momentum map to $P_{g^{*}}$ and dropping down to $J g^{*}$.

The process of reduction commutes with taking the path space or jet space. The jet or path space of the reduced space is the reduced space of the jet or path space by the jet or path group.

We have seen the central importance of the dual of the Lie algebra and the coadjoinit orbits with their KKS symplectic structure for physics. We have seen that any symplectic manifold may be thought of as a coadjoint orbit in the dual of the Lie algebra of some group. It turns out that if $M$ is a coadjoint orbit in the dual of $G$ 's Lie algebra then the perturbation space $J M$ with the jet symplectic structure are naturally a coadjoint orbit in the dual of the Lis algebra of the jet group $J G$ and the jet bracket (51) is the natural KKS symplectic structure.

These relations are at the heart of a new framework for singular Lie transform perturbation theory about which we will reprort elsewhere. Here we discuss only the first order method of averaging.

\section{The Method of Averaging for Hamiltonian Systems}

Many of the interesting physical regularities we find in diverse systems are caused by the presence of processes that operate on widely separated time scales. The basic simplification this entails is that the fast degrees of freedom act almost as if the slow variables are constant and the slow degrees of freedom are affected only by the average behavior of the fast variables. Bogoliubov in particular has used this separation of scales with great success in many examples. For example he obtains the Boltzmann equation from the BBGKY hifrarchy of evolution equations for correlation functions by holding the 1-particle distribution functions fixed while determining the fast evolution of the higher correlations, and then substituting the result in as the collision term driving the 1-particle evolution. One makes a similar separation in calculating fluid quantities like viscosity, thermal conductivity, diffusion or electrical conductivity from an underlying kinetic description. In studying complex situations with slow, isiaty nuclei and fast, light electrons in 
molecular and solid state physics, one often holds the nuclei fixed, calculates the electron ground state and energy as a function of the nuclei positions and then uses them to define an effective potential in which the nuclei move. We have seen that in the presence of an exact symmetry, the symmetry directions may be completely eliminated by the process of reduction. One often finds that the effect of "forgetting" these degrees of freedom is to introduce an amended potential into the Hamiltunian and a "magnetic" piece to the Poisson bracket of the reduced system. We have the centrifugal force coming out as an effective potential earlier. We will report on a version of this reduction procedure which begins by including the "angle of the earth" as a dynamical variable and reduces by the earth's rotation and the rotation of the system together. The resulting reduced space gives the centrifugal force as an amended potential in the reduced Hamiltowian and the Coriolus force as a new term in the Poisson bracket.

When we introduce a perturbation which breaks a symmetry we no longer have exactly conserved quantities. It is easy to prove an "approximate Noether's theorem", however, which says that the momentum map for a slightly broken symmetry evolves slowly:

$$
X_{J} \cdot H=\{H, J\}=\epsilon \quad \text { implies } \quad j=\{J, H\}=-\epsilon .
$$

In the special case where the unperturbed dynamics is entirely composed of periodic orbits, the action of the orbit through each point is the momentum map of a circle symmetry of the unperturbed Hamiltonian. As we turn on a perturbation which breaks this symmetry, the motion will still be primarily around the loops, but it will slowly drift from loop to loop. Because the symmetry is broken, different points on a loop will move toward different loops. As the perturbation is made smaller, though, phase points orbit many times near a given loop before drifting away. This suggests (correctly) that the perturbation a point feels will asymptotically be the same as the average around an unperturber loop. Because this average is the same for all points on a loop, for small perturbations entire loops drift onto other entire loops. We may therefore drop the dynamics down to the loop space. In fact one can prove that for a general (even dissipative) system where the unperturbed dynamics $X_{0}$ is entirely composed of periodic orbits, the motion of a point under the flow of $X_{0}+\varepsilon X_{1}$ projected down to the loop space remains within $\epsilon$ for a time $1 / \epsilon$ of the orbit of a corresponding point on the loop space under the flow of the average of $X_{1}$ around each loop projected down ${ }^{35}$. In the Hamiltonian case we break the circle symmetry of $H_{0}$ to get the perturbed system $H_{0}+H_{1}$. We average $H_{1}$ around the loops to get $\bar{H}_{1} . H_{0}+\bar{H}_{1}$ is again invariant under the circle acticn and so we may perform reduction. The reduced dynamics is the slow dynamics on the reduced space and the fact that we may restrict to a constant value of the momentum map, shows that it is actually conserved to within order $\epsilon$ for time 1/c. This is because loops are taken to loops to this order and the action of a loop (i.e. the integral of the symplectic form $\omega$ over a sheet whose boundary is the loop) is left invariant under a canonical transformation (like the flow of the perturbed system) since $\omega$ is. Kruskal has shown that there is actually a quantity which is conserved to all orders in $\epsilon$ for time $1 / \epsilon^{36}$ (we will report on a geometric formulation of this result in a future paper). Getting results valid for times longer than $1 / \epsilon$ is extremely important physically, but so fa: no general theory exists. 
Let us relate this procedure to the perturbation structures we developed in previous sections. We have an action of the circle group $S^{1}$ on $M$. This lifts to an action of $P S^{1}$ on $P M$ and $J S^{1}$ on $J M$. The unperturbed Hamiltonian is invariant under the $S^{1}$ action on $M$, but the path and perturbation Hamiltonians are not invariant under $P S^{1}$ and $J S^{1}$. We would like to change the action of $P S^{1}$ on $P M$ so as to leave the path Hamiltonian invariant and so allow reduction. Since the resulting action should still be Hamiltonian, we look for an $\epsilon$-dependent canonical transformation of $I \times M$ which is the identity at $\epsilon=0$ and which pushes the $P S^{1}$ action into a symmetry. The method of Lie transforms attempts to do this at the perturbation level, letting the canonical transformation be the flow of an e-dependent Hamiltonian, which is then obtained order by order. Here we need only consider the first order action of $1 S^{1} \sim T S^{1}$ on $1 M \sim T M$. We know that the action will be perturbed so that the value of the reduced Hamiltonian is the ayerage of the perturbed Hamiltonian around the untransformed circles. $T M$ has twice the dimension of $M$. Reducing by $T S^{1}$ eliminates 4 dimensions. The resulting dynamical vector field has no unperturbed component. One may think of this as the reason for getting results good for time $1 / \epsilon$ (it is the action of the unperturbed flow on the perturbation which causes this level of secularity). In this situation it makes intrinsic sense to project the 1 st order vector tield cown to $M$, where it represents the slow dynamics.

A loop in a 2-dimensional plase space (like an orbit of a simple harmonic oscillator) may be thought of in 3 ways. It is 1-dimensional, 1 dimension less than 2, and half of 2 . Each has an important generalization to higher dimensional Hamiltonian systems. In the presence of a slowly varying Hamiltonian, we have already seen that the action of a 1-dimensional loop is conserved. There is an analogous result for half dimensional Lagrangian tori. Kubo ${ }^{37}$ has shown that for a system ergodic o $\alpha$ an energy surface (which has one dimension less than phase space), the volume exclosed is adiabatically invariant under slow variation of parameters. Roughly: since the motir $\mathbf{a}$ is ergodic, every orbit changes according to the average of the perturbation over the energy surface; thus the entire energy surface changes by the same energy and so is taken to another energy surface; but the volume enclosed by a surface is preserved under a canonical transformation by Liouville's theorem. For a large number of degrees of freedom this leads to the adiabatic invariance of the entropy in statistical mechanics.

The funny potentials and Poisson brackets that result from reduction contain the average effect of the fast on the slow degrees of freedom. Capturin ${ }_{B}$ this effect is the content of many physically useful theories. It is interesting to nots that in the late eighteenth century, the idea that all potential energies were really kinetic energies of hidden or forgotten degrees of freedom was one the the main motivations for the development of kinetic theory. We may use averaging to see how this comes about. If we slowly move a ping pong paddle up and down from a.table with a ping pong ball bouncing very rapidly between the paddle and the table, then we will feel a varying force due to the average momenta imparted due to the impacts of the ball. In phase space the ball describes a rectangle and so the action is given by $J=4 L \mathrm{mV}$ where $\mathrm{L}$ is the distance from the paddle to the table and $V$ is the speed of the ball. Because this is invariant under slow paddle movements, the ball velocity goes as $1 / L$. The momentum transferred on each impact is 
$2 m V$ and there are $V / 2 L$ impacts per unit of ime, so the average force felt goes like $V^{2} \sim 1 / L^{2}$. Thus starting with no potential energy at all, we end up with a $1 / L$ effective potential for the paddle!

For a harmonic oscillator, the energy is the product of the action and the frequency: $H=\omega J$. If we have a weight hanging on string undergoing snall amplitude oscillations as we siowly pull the string, the change in pendulum energy is the change in $J \omega . J$ remains constant and $\omega \sim \sqrt{g / L}$ so we feel a $1 / \sqrt{L}$ potential. We get other potentials if we ask for the force we feel if we tune a guitar string as someone plays it or the acoustic pressure on the water if we fill up a shower. as someone sings in it. The effective force due to the fast degrees of freedom may sometimes stabilize an unstable fxed point of the slow system. Ordinarily an inverted penduium is unstible and falls to the position with the weight hanging downward. If we shake the support of the pendulum periodically hard enough and fast enough, the inverted position is stabilized! An even more sfectacular version of this effect occurs if you shake an inverted cup of fluid and stabilize the Rayieigh-Taylor instability which ordinarily causes the fluid to spill out (it is easiest to actually do the experiment with a high viscosity fluid like motor oil). The idea of RF stabilization is to stabilize unstable modes of a plasma (say in a tolamak) by bathing it in a high frequency radio wave. Some of the modern airplanes with wings in a forward facing delta are actually operated in an aerodynamically unstable regime that is stabilized by the fast dynamics of a computer controlled feedback loop. This allows for great maneuverability (since the plane would like to turn anyway!).

Quite often it is very usefull to split out the main dynamics of a system and linearize the rest, treating them as fast oscillations. Thus one takes a fluid, elastic, or plasma medium and treats its evolution as slow overall development of the background medium with fast oscillations occuring on top of it. The effect of the oscillations is to change or renormalize the dynamics of the background. N.G. van Kampen ${ }^{40}$ has called into question the usual treatments of constrained mechanical systems. One usually just writes down the Lagrangian for such a system in generalized coordinates which respect the constraints. Physically, though, one supposes that there is some large potential nurmal to the constraint surface. The system will execute rapid oscillation in the normal direction and slow evolution along it. If the width of the constraining potential well varies with the mechanical coordinates, then as we have seen the adiabatic invariance will give rise to a new pseudopotential which affects the mechanical motion. In a plasma we treat the siowly varying background as a dialectric medium in which waves propagate according to WKR theory. The waves affect the background (introducing a radiation pressure in the dynamics) via pondermotive forces. If we have a charged particle in the presence of a wave with a slowly varying amplitude, the particle will oscillate back and forth with the wave. It feels more of a push in going down an amplitude gradient than in going up one, leading to an overall average force described by the pondermotive potential. This kind of separation is the basis of plasma quasilinear theory. We have extended the geometric perturbation theory to some of these singular perturbation problems. We will report elsewhere on a Hamiltonian treatment of an eikonal theory for linear or nonlinear waves (which is related to the averaged Lagrangian treatment of Whitham ${ }^{38}$ ). Here let 
us demonstrate the efficacy of a global geometric approach only with the simple example of $E \times B$ drift. A particle in a constant magnetic field executes perfect circles. If there is, in addition, an electric field then the radius of the circles is greater in low potential regions and smaller in high potential regions. Thus the circular orbits do not close and the particle dritits perpendicularly to the electric field. A Hamiltonian treatment of more complicated versions of this so-called guiding center motion has been previously given ${ }^{39}$. This work required great cleverness in the choice of physically relevant coordinates. We would like to demonstrate, in this siraple version, how a coordinate free approach would lead us to the correct answer, with no previous knowledge.

\section{Example: $E \times B$ Drift}

In the simplest situation we have a charged particle in the $x, y$ plane moving in the presence of a constant magnetic field $B$ which points in the $\hat{z}$ direction and a small constant electric field $E$ which points in the $\hat{x}$ direction. We introduce the phase space $P \sim T^{*} R^{2}$ with coordinate: $\left(x, y, p_{x}, p_{y}\right)$ (we use mechanical momenta $p=m v$ here). The correct dynamics in the presence of a magnetic field may be described in a Hamiltonian formulation in two ways. The standard approach is to introduce the unphysical vector potential $A$ and to work with canonical momenta $p=m v-(e / c) A$. Here we use the physical momenta and magnetic field, but a noncanonical Poisson bracket:

$$
\{f, g\}=f_{x} g_{p_{x}}-f_{p_{x}} g_{x}+f_{y} g_{p_{y}}-f_{p_{y}} g_{y}+\frac{e B}{c}\left(f_{p_{x}} g_{p_{y}}-f_{p_{y}} g_{p_{z}}\right)
$$

We obtain the correct dynamics in this case with the Hamiltonian:

$$
H=H_{0}+\epsilon H_{1}=\frac{1}{2 m}\left(p_{x}^{2}+p_{y}^{2}\right)-\epsilon e E x .
$$

The dynamics is then:

$$
\begin{aligned}
\dot{x} & =\frac{p_{x}}{m} \quad \dot{y}=\frac{p_{y}}{m} \\
\dot{p}_{x} & =\frac{e B}{m c} p_{y}+\epsilon e E \quad \dot{p}_{y}=-\frac{e B}{m c} p_{x}
\end{aligned}
$$

The unperturbed situation here is just a charged particle on a plane in a constant magnetic field. Every orbit in this situation is a closed loop. Thus the unperturbed system has a circle symmetry:

$$
\begin{gathered}
\dot{x}=\frac{p_{x}}{m} \quad \dot{y}=\frac{p_{y}}{m} \\
\dot{p}_{x}=\frac{e B}{m c} p_{y} \quad \dot{p}_{y}=-\frac{e B}{m c} p_{x}
\end{gathered}
$$

The generator of this symmetry (i.e. the momentum map) is none other than the unperturbed Hamiltonian itself:

$$
H_{0}=\frac{1}{2 m}\left(p_{x}^{2}+p_{y}^{2}\right)
$$

Let us obtain the reduced phase space and Poisson bracket for this symmetry action. First we look at the space of loops $P / S^{1}$. Each circular particle orbit has exactly one point where $p_{y}=0$ 
and $p_{x} \geq 0$. We may label a loop by the values of $x, y, p_{x}$ at this point. Next we restrict to the set where the momentum map is a constant: $H_{0}=\alpha$. The reduced space is

$$
R=P /\left.S^{1}\right|_{H_{0}=\alpha}
$$

and may be coordinatized by the values of $x$ and $y$ when $p_{x}=\sqrt{2 m \alpha}$ and $p_{y}=0$. The reduced Poisson bracket $\{,\}_{\alpha}$ of two functions $f(x, y)$ and $g(x, y)$ is obtained by extending them to $\mathrm{P}$ in such a way that:

$$
\left.\frac{\partial \hat{f}}{p_{x}}\right|_{p_{x}=\sqrt{2 m \alpha}, p_{y}=0}=0
$$

and

$$
\left\{\hat{f}, H_{0}\right\}=0=\frac{\sqrt{2 m \alpha}}{m} \frac{\partial \hat{f}}{\partial x}-\frac{e B}{m c} \sqrt{2 m \alpha} \frac{\partial \hat{f}}{\partial p_{y}}
$$

Thus we replace $\partial / \partial p_{x}$ by 0 and $\partial / \partial p_{y}$ by $(c / e B) \partial / \partial x$ to get:

$$
\{f, g\}_{a r}=\frac{c}{e B}\left(f_{y} g_{x}-f_{x} g_{y}\right) \text {. }
$$

Thus we see that the original spatial coordinates $x$ and $y$ now play the role of canonically conjugate variables in the reduced space. The factor of $1 / B$ in the bracket appeared in Littlejohn's work ${ }^{39}$. The full system is not invariant under our circle action. If we average the perturbation Hamiltonian $H_{\mathrm{I}}$ around the circles, we do obtain a circle symmetric system. The average of the potential $\epsilon e E x$ around a loop is just the value when $p_{y}=0$. Thus the reduced averaged Hamiltonian is:

$$
\bar{H}_{\alpha}(x, y)=\alpha-\epsilon e E x .
$$

The reduced averaged dynamics is then:

$$
\begin{aligned}
& \dot{x}=\left\{x, \bar{H}_{\alpha}\right\}_{\alpha}=0 \\
& \dot{y}=\left\{y, \bar{H}_{\alpha}\right\}_{\alpha}=\frac{c}{e B}(-\epsilon e E)=-\epsilon \frac{c E}{B}
\end{aligned}
$$

This is indeed the $E \times B$ drift dynamics.

\section{Acknowledgemente}

I would like to thank Ted Courant, Allan Kaufman, Robert Littlejohn, Jerry Marsden, Rich Montgomery, and Alan Weinstein for many suggestions and discussions regarding these ideas. This paper was typeset on computer using $\mathrm{T}_{\mathrm{E}} \mathrm{X}$.

\section{Bibliography}

[1] J. L. Lagrange, "Mémoire sur la théorie des variations de éléments des planètes",Mémoires de la classe des sciences mathématiques et physiques de l'instiut de France (1808), pp.1-72. 
[2] A. Weinstein, "Symplectic Geometry", Bulletin of the American Mathematical Society 5 (1981), 1-13.

[3] H. Poincaré, Les méthodes nouvelles de la mécanique céleste, 1,2,3. Gauthier-Villars, Paris (1892); Dover, New York, 1957.

[4] E. Noether, Nachrichten Gesell. Wissenschaft. Gottingen 2 (1918), 136.

[5] P. A. M. Dirac, The Principles of Quantum Mechanics, Oxford University Press, 1958.

[6] As in L. D. Landau and E. M. Lifshitz, Course of Theoretical Physics, Volumes 1-10, Pergamon Press Ltd., New York (1960-1981).

[7] R. Abraham and J. E. Marsden, Foundations of Mechanics, 2nd edition, Benjamin/ Cummings, Reading, Mass., 1978.

[8] V. I. Arnold, Mathematical Methods of Classical Mechanics, Graduate Texts in Math., vol. 60, Spriuger-Verlag, Berlin and New York, 1978.

[9] J. R. Cary, "Lie Transform Perturbation Theory for Hamiltonian Systems",Physics Reports 79 (1981), 131.

[10] P. R. Chernoff and J. E. Marsden, Properties of Infinite Dimensional Hamiltonian Systems, Lecture Notes in Math., vol. 425, Springer-Verlag, New York (1974).

[11] P. J. Morrison and J. M. Greene, "Noncanonical Hamiltonian Density Formulation of Hydrodynamics and Ideal Magnetohydrodynamics", Phys. Rev. Letters 45 (1980), 790-794.

[12] J. E. Marsden and A. Weinstein, "Coadjoint Orbits, Vortices, and Clebsch Variables for Incompressible Fluids", Physica 7D (1983), 305-323.

[13] J. E. Marsden, T. Ratiu, and A. Weinstein, "Semi-direct Products and Reduction in Mechanics", Transactions of the American Mathematical Society 281 (1984), pp. 147-177.

[14] P. J. Morrison, "The Maxwell-Vlasov Equations as a Continuous Hamiltonian System"; Phys. Lett. 80A (1980), 383-396.

[15] J. Marsden and A. Weinstein, "Tne Hamiltonian Structure of the Maxwell-Vlasov Equations", Physica 4D (1982), 394-406.

[16] W. Pauli, General Principles of Quantum Mechaoics, (1933) Reprinted in English Translation iy Springer-Verlag, Berlin, 1981.

[17] D. Holm and B. Kupershmidt, "Poisson Brackets and Clebsch Representations for Magnetohydrodynamics, Multifluid Plasmas, and Elasticity", Physica D.

(18) J. E. Marsden and T. J. R. Hughes, Mathematical Foundations of Elasticity, Prentice-Hall, Englewood Cliffs, New Jersey, 1983.

[19] R. G. Spencer and A. N. Kaufman, "Hamiltonian Structure of Two-Fluid Plasma Dynamics", Phys. Rev. A 25 (1982) 2437-2439.

[20] J. Gibbons, D. D. Holm, and B. Kupershmidt, "Gauge-Invariant Poisson Brackets for Chromohydrodynamics", Physics Letters 90A (1982), 281-283.

[21] D. D. Holm and B. A. Kupershmidt, "Poisson Structures of Superfluids", submitted to Physics Letters A.

[22] L. Faddeev and V. E. Zakharov, "Korteweg-de Vries as a Completely Integrable Hamiltonian System”, Funct. Anal. Appl. 5 (1971) 280. 
[23] D. D. Holm, J. E. Marsden, T. Ratiu, and A. Weinstein, "A Priori Estimates for Nonlinear Stability of Fluids and Plasmas ${ }^{n}$, preprint, (1984).

[24] V. Guillemin and S. Sternberg, Symplectic Techniques in Physics, Cambridge University Press, Cambridge, 1984.

[25] S. Omohundro, "Hamiltonian Structures in Perturbation Theory", submitted to the Journal of Mathematical Physics (1984).

[26] S. Omohundro, "A Hamiltonian Approach to Wave Modulation", Poster presented at April 1984 Sherwood Plasma Theory Meeting, Lake Tahoe, in preparation for publication.

[27] A. H. Nayfeh,Perturbation Methods, John Wiley and Sons, Inc., New York, 1973.

[28] J. Kevorkian and J. D. Cole, Perturbation Methods in Applied Mathematics, Springer-Verlag, New York, 1981.

[29] A. Weinstein, "The Local Structure of Poisson Manifolds" , J. Differential Geometry 18 (1983) 523-557.

[30] J. E. Marsden, Lectures on Geometric Methods in Mathematical Physics, CBMS-NSF Regional Conference Series 37, Philadelphia, PA:SIAM, 1981.

[31] P. Olver, "Hamiltonian perturbation theory and water waves", in J. Marsden, ed., Fluids and Plasmas: Geometry and Dynamics, American Mathematical Society series in Contempcrary Mathematics, Volume 28, 1984, p. 231.

[32] J. Marsden and A. Weinstein, "Reduction of symplectic manifolds with symmetry", Rep. Math. Phys. 5 (1974) 121-130.

[33] V. Arnold, "Sur la geometrie differentielle des groupes de Lie de dimension infinie et ses applications a lhydrodynamique des fluids parfaits", Ann. Inst. Fourier Grenoble 16(1) (1966) 319-361.

[34] J. Kijowski and W. Tulczyjew, A symplectic framework for feld theories, Lecture Notes in Physics 107, Springer, New York, 1979.

[35] V. Arnold, Geometrical Methods in the Theory of Ordinary Differential Equations, SpringerVerlag, New York, 1983.

[36] M. Kruskal, "Asymptotic theory of Hamiltonian and other systems with all solutions nearly periodic", J. Math. Phys. 3, (1962) 806-828.

[37] R. Kubo, H. Ichimura, T. Usui, N. Hashitsume, Statistical Mechanics, North-Holland, Amsterdam, 1965.

[38] G. Whitham, Linear and Nonlinear Waves, John Wiley \& Sons, Inc., New York, 1974.

[39] R. Littlejohn, J. Plasma Physics 29 (1983) 111.

[40] N. van Kampen, "Constraints", preprint, March 1983. 
- This reporn was done with support from the Eipartment of Energy. Any conslusions or opinions expressed in this report represent solely thase of the author(3) and not necessarily those of The Regents of the University of Californiz, the Lawrence Berkeley Leboralory of the Depanment of Energy.

Reference to a company or product name does not imply approval or recommendation of the product by the University of Coliforniz or the U.S. Department of Energy to the exclusion of others that may be suitable.

i: 